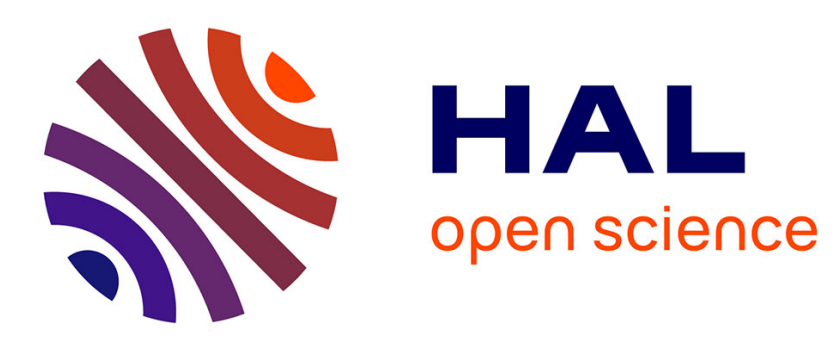

\title{
Effect of Tear Rotation on Ultimate Strength in Reinforced Natural Rubber
}

Brice Gabrielle, Laurent Guy, Pierre-Antoine Albouy, Loïc Vanel, Didier R.

Long, Paul Sotta

\section{- To cite this version:}

Brice Gabrielle, Laurent Guy, Pierre-Antoine Albouy, Loïc Vanel, Didier R. Long, et al.. Effect of Tear Rotation on Ultimate Strength in Reinforced Natural Rubber. Macromolecules, 2011, 44 (17), pp.7006 - 7015. 10.1021/ma2010926 . hal-01727367

\section{HAL Id: hal-01727367 https://univ-lyon1.hal.science/hal-01727367}

Submitted on 16 Mar 2018

HAL is a multi-disciplinary open access archive for the deposit and dissemination of scientific research documents, whether they are published or not. The documents may come from teaching and research institutions in France or abroad, or from public or private research centers.
L'archive ouverte pluridisciplinaire HAL, est destinée au dépôt et à la diffusion de documents scientifiques de niveau recherche, publiés ou non, émanant des établissements d'enseignement et de recherche français ou étrangers, des laboratoires publics ou privés. 


\title{
Effect of tear rotation on ultimate strength in reinforced natural rubber
}

\author{
Brice Gabrielle ${ }^{1}$, Laurent Guy ${ }^{2}$, Loïc Vanel ${ }^{1,3}$, Didier Long ${ }^{1}$, Paul Sotta ${ }^{1 *}$ \\ 1 Laboratoire Polymères et Matériaux Avancés (LPMA), \\ CNRS/Rhodia UMR 5268 \\ CRTL \\ 85 rue des frères Perret \\ 69192 Saint-Fons Cedex, France \\ 2 Rhodia \\ 3 Laboratoire de Physique de la Matière Condensée et Nanostructures (LPMCN) \\ CNRS/Université Lyon 1 UMR 5586 \\ Villeurbanne, France
}

$16 / 03 / 2011$

\begin{abstract}
Vulcanized natural rubber samples reinforced with carbon black or precipitated silica are studied. Single edge notched reinforced samples stretched at constant velocity exhibit an abrupt instability in the direction of propagation of the crack. This phenomenon has been known as tear rotation. We analyze the impact of tear rotation on the tensile strength of the samples. A mechanism is proposed to relate the presence of tear rotations observed in reinforced natural rubber to the tensile strength of the material. The measured apparent tensile strength (in terms of energy at break) may be increased by a factor of 6 to 8 in some cases. This large increase in tensile strength associated to the presence of tear rotations is analyzed semi-quantitatively, based on energetic arguments, without entering into a detailed description of the elastic strain field in the vicinity of the tear tip. We also show a correlation between the length of a rotation and the stress level at which it appears. The proposed interpretation is based on the idea that tear rotations relax the local strain (or stress) at the tear tip by creating a macroscopic tip radius. Materials reinforced with carbon black or precipitated silica aggregates show similar behavior.
\end{abstract}




\subsection{Introduction}

Various instabilities are observed as a crack propagates in a reinforced rubber. In trouser tests as well as in pure shear tests, so-called "knotty tearing" is observed ${ }^{1,2}, 3$. In single-edge-notched tension samples (SENT) submitted to tensile loading at constant velocity, cracks tend to rotate in a direction perpendicular to the usual one, i.e. parallel to the applied tensile stress ${ }^{4}$. In this case, it has been called 'hammer head' cracking ${ }^{5}$ or 'tear rotation'. Instabilities in the direction of propagation have been quite extensively documented in carbon black (CB)-filled natural rubber $(N R)^{4},{ }^{4}, 7$, as well as in some cases, in CB-filled styrene-butadiene rubbers (SBR) ${ }^{7}$.

Tensile specimens with razor cut notches of different lengths have been used to characterize resistance to tear propagation of various rubber and reinforced rubber materials ${ }^{48,9}$. It was observed that pre-cut, filled NR samples show a much higher resistance to cut growth as compared to SBR samples, and that this is associated to instabilities of the direction of tear propagation $4,10,11$, $12,13,14$. The effect of various material parameters, such as the crosslink density ${ }^{9},{ }^{15}$ and the volume fraction ${ }^{16}$ and reinforcing power of fillers, was studied. It was already suggested that strain-induced crystallization may prevent mode I (i.e. perpendicular to the load direction) growth of the original cut notch in NR samples ${ }^{17},{ }^{18}$. It was observed that the relation between tensile strength (or stress at break) and pre-cut notch length is not simple in the presence of tear rotation. Various regimes of variation seem to occur in various ranges of cut length. A threshold value $c_{c r} \approx 2 \mathrm{~mm}$, above which the tensile strength drops quite abruptly, seems to be observed ${ }^{9}, 16$. This was interpreted as corresponding to the onset of strain-induced crystallization in the bulk of the test sample. This assumption will be discussed later in this paper. In some cases, it was observed that the tensile strength of carbon-black reinforced materials is reduced as compared to the pure gum ${ }^{16}$.

Description of crack propagation in rubbery materials is faced with two main difficulties: rubber materials can sustain extremely large elastic deformations (up to several 100\%), and the viscoelastic contribution to tearing energy (dissipation) may be significant, or even perhaps dominant, in some regimes of interest, as it is evidenced by the large variation of the tearing energy with the tear propagation rate ${ }^{19,20}$. Moreover, dissipation is eventually not localized in a very small region close to the crack tip but may occur in a very large (macroscopic) region of the material.

At a macroscopic scale, the first approach which has been developed to analyze fracture toughness of a material is based on energetic balance arguments ${ }^{21}$. Here we briefly recall some basic arguments of this approach. The driving force for propagation is the energy release rate, i.e. the elastic energy recovered when a unit crack length is created by crack propagation at constant overall deformation of the test sample, denoted $G$ in the following:

$$
\text { 1. } G=-\frac{1}{e}\left(\frac{\partial U_{e l}}{\partial c}\right)_{\lambda}
$$

where $e$ is the sample thickness, $U_{e l}$ is the elastic energy stored in the sample, $W$ the elastic energy density within the uniformly deformed part of the material (far away from the crack) and $\lambda$ is the elongation ratio in this same part of the material, or more precisely, this would be the elongation ratio in an un-notched sample of same overall elongation. 
In the case of single-edge-notched tensile (SENT) samples, it was shown that the energy release rate $G$ is, for a crack (precut notch) of length $c$, in the case of normal propagation, i.e. without instability in the direction of propagation ${ }^{22,5}$ :

$$
\text { 2. } G \cong 2 \pi \frac{W c}{\sqrt{\lambda}}
$$

A crack of length $c$ becomes unstable when the energy release rate (equation 2 ) becomes equal to the tear energy $\Gamma$, which may be considered as a characteristic property of the material. If this instability threshold is identified with the failure of the test sample, then the following relationship between the elastic energy density stored in the material at failure $W_{b}$ (energy at break) and the precut notch length $c$ is obtained:

$$
\text { 3. } \frac{W_{b}}{\sqrt{\lambda_{b}}}=\frac{\Gamma}{2 \pi c}
$$

Note that, in the case of normal propagation, according to equation 2, the energy release rate increases as the crack length $c$ increases. Thus, in the chosen experimental configuration (singleedge-notched tension strips (SENT samples) of finite width stretched at a given elongation rate), there is no regime of stable, slow crack growth. As a consequence, this experimental geometry does not allow controlling the crack growth rate and thus is not appropriate to characterize the tear energy vs crack growth rate curve.

In this paper, we show measurements of the tensile strength of SENT samples stretched at constant velocity. Only single propagation experiments, as opposed to cyclic (fatigue) experiments, are considered. Samples with different rubber matrices (SBR, NR), and different reinforcing systems (carbon black, silica with covalent coupling to the rubber matrix) are compared. We find that only reinforced NR materials exhibit tear rotation and that tear rotations have a large impact on the tensile strength of the samples. We relate the energy at break (i.e. the elastic energy which is stored in the material before it breaks) measured in pre-notched tensile test samples to the overall crack contour length (which takes into account tear rotation). We discuss some features of tear rotations in terms of energetic arguments. We show that it is possible to draw certain conclusions on tear rotations and on their effect on the measured tensile strength of samples, based on energy balance arguments.

The proposed arguments may perhaps be used to predict the resistance to tear of various reinforced natural rubber materials (or perhaps other materials exhibiting tear rotation as well), based on the observation and analysis of the tear rotation patterns.

\section{Materials and methods}

In order to compare and discuss the influence of the rubber matrix and of the reinforcing filler system on tear propagation properties, six sample formulations have been used, with two types of rubber matrices (Styrene butadiene rubber (SBR) and Natural Rubber (NR)) and two types of reinforcing fillers (carbon black and precipitated silica). Details of sample formulations are given in

Table 1. In silica-filled samples, a coupling agent (bis triethoxysilylpropyl tetrasulfur, (TESPT)), which provides covalent bonding of the NR matrix to the silica surface, has to be used to obtain good reinforcement properties. Both reinforcing systems (carbon black or silica plus coupling agent) 
provide the same hardness (60 Shore A) and similar reinforcement properties at the same volume fraction (about 20 vol\%) in both rubber matrices (see Figure 1 ).

All experiments were carried out on a MTS $1 /$ Me tensile apparatus with self-tightening grips and a long range mechanical extensometer using rectangular strip pieces $\left(12 \times 70 \times 2 \mathrm{~mm}^{3}\right)$. Single edge notched samples are precut to a length varying between roughly 0.3 and $4 \mathrm{~mm}$. Samples were stretched at a controlled, constant velocity, varying between $5 \mathrm{~mm} / \mathrm{min}$ (tensile strain rate $1.4 \times 10^{-3}$ $\mathrm{s}^{-1}$ ) and $500 \mathrm{~mm} / \mathrm{min}$ (tensile strain rate $0.14 \mathrm{~s}^{-1}$ ). A pre-stress of $0.05 \mathrm{MPa}$ was applied before starting the experiments. Experiments shown here were performed with as prepared samples, without precycling. The elastic energy density $W$ is obtained by integrating the force-extension curve and dividing by the volume of the stretched sample. Note that, in precut samples, this gives only an approximation of the actual energy density stored in the material far from the tear, since in that case the strain is not uniform throughout the sample. We have chosen to use as a parameter suitable to describe tensile strength of samples the elastic energy density at the ultimate breaking point $W_{b}$ measured for SENT samples precut at various cut lengths. Using notched samples ensures that failure will occur at a given predetermined location (the tip of the precut notch). Thus each sample can be characterized by a characteristic relationship between the precut notch length $c$ and the energy density at break $W_{b}(c)$.

Video monitoring of crack propagation was done with an IMPERX 16M3 camera. The camera was moved at half the crosshead speed in order for the crack to remain in the image field during the tensile test. Image and data acquisitions were synchronized.

Scanning electron microscope (SEM) observations were performed with a Zeiss SEM. The surface of the samples was metalized with platinum. Rotation lengths are measured by image analysis of SEM observations with a MatLab routine.

\section{Results}

\subsection{Estimating the tearing energy}

We first characterize the behaviour of unnotched samples $(c=0)$ in a tensile test performed at drawing speed 50 $\mathrm{mm} / \mathrm{min}$ (deformation rate $\dot{\varepsilon}=1.4 \times 10^{-2} \mathrm{~s}^{-1}$ ). The corresponding nominal stress vs strain curves in the studied samples (unnotched strip samples) are shown in Figure 1. End points of the curves correspond to failure of the samples. These curves show that the reinforcement provided by either carbon black or silica, at the same volume fractions, are comparable in the studied formulations, even though stress-strain curves show significant differences in their shapes at large strain. Pure NR breaks at much larger strain and stress than SBR. It is well known that enhanced ultimate properties of pure NR, as compared to pure SBR, are due to strain induced crystallization, which occurs around $400 \%$ strain. In filled SBR samples, both the stress and strain at break are increased compared to pure SBR. In filled NR samples, only the stress at break increases while the strain at break decreases upon addition of fillers. Nevertheless, the ultimate stress and strain of filled NR samples remain both significantly higher than for filled SBR samples. From the stress-strain curves in Figure 1, the energy density at failure $W_{f}$ can be measured on uncut samples $\left(W_{f}=W_{b}(c=0)\right)$. This quantity characterizes the samples at a macroscopic scale. Its value (column 4 in

Table 2) is distinct from the energy at break measured in notched test samples.

Next, we have measured the elastic energy density at break $W_{b}$ of single edge notched tensile test (SENT) samples as a function of the precut notch length $c$, at room temperature. It is observed that slow forward propagation of the crack, if any, occurs on a very small length scale, just before fast, catastrophic failure. This slow propagation step therefore contributes only negligibly to the overall 
energy at break. In Figure 2, the quantity $\frac{W_{b}}{\sqrt{\lambda_{b}}}$ is plotted as a function of $c$, according to equation 3. The results obtained in reinforced NR samples are compared to those obtained in reinforced SBR samples. Data for SBR may be reasonably fitted according to equation 3, which gives a tear energy $\Gamma_{\mathrm{SBR}}=15.7 \mathrm{~kJ} \cdot \mathrm{m}^{-2}$. Reinforced NR samples have a resistance to tear propagation (as expressed by the energy density at break $W_{b}$ ) typically 3 to 15 times higher than reinforced SBR. Also remarkable is the very large scattering of the reinforced NR data compared to SBR data.

The same is observed when data for reinforced NR samples are compared to pure (unfilled) NR samples (Figure 3). In this case, energy densities at break in reinforced NR are 1.5 to 8 times larger than in pure NR samples. Again, the data for pure NR are compatible with equation 3 (though in a rather limited range of precut notch length $c$, typically larger than $0.6 \mathrm{~mm}$ ), which gives a tearing energy $\Gamma_{\mathrm{NR}}=31.4 \mathrm{~kJ} . \mathrm{m}^{-2}$ (see Figure 3 ).

Based on the results in Figure 2 and Figure 3, it cannot be concluded that the increased tensile strength (or average resistance to tear propagation) observed in reinforced NR as compared to pure NR and reinforced SBR may be attributed to an intrinsically higher tear energy $\Gamma$. Indeed, equation 3 does not take tear rotation into account, which provides a new mechanism of tear propagation not observed in reinforced SBR and in pure NR. Also, fitting the data for reinforced NR in Figure 2 or Figure 3 with equation 3 using a higher $\Gamma$ value would not account for the large scattering of the data. Actually, when only small rotations are developed in some filled samples, the energy at break is very close to that in pure NR (see Figure 3), which indicates that the observed increase in tensile strength should rather be attributed to tear rotations than to an intrinsically higher tear energy. Despite the scattering of the reinforced NR data, Figure 3 suggests that NR samples have an intrinsically higher tearing energy $\Gamma$ than reinforced SBR samples. The effect of tear rotation is quantitatively discussed in the next section.

From Figure 2 and Figure 3, it is very difficult to discriminate the samples according to the nature of the reinforcing system: samples reinforced with carbon black (cross-filled square symbols) or silica (empty squares) give widely overlapping clouds of representative points.

\subsection{Tear rotation}

The tear (initiated at the pre-cut notch) propagates in different ways in the various samples. In pure NR as well as in both pure and reinforced SBR samples, tear propagation is perpendicular to the drawing direction, as expected. Propagation occurs in a fast, catastrophic way, with relatively smooth fracture surfaces. In reinforced NR samples, tear propagation is more complex, with a well-developed tear rotation phenomenon. Both CB- and silica-filled NR samples qualitatively show the same behavior, in which three steps may be distinguished (Figure 4). First, the pre-cut notch opens without significant propagation, up to a point at which the tear tip is widely open down to the micrometer scale. The second step is the occurrence of rotations. A tear initiates in the central region of the widely open tip, propagates perpendicularly to the tensile direction, over a small distance (typically a few tens to a few 100s of $\mu \mathrm{m}$ ), and then rotates abruptly on both sides along the direction of the applied force. This leads to the appearance of two rolls or folds of relaxed material curling along the crack lips in the tensile direction. Propagation along the tensile direction is slow and stops at some point. Then, as the applied load increases, a second tear eventually initiates somewhere along the flat crack tip and eventually rotates again. The whole process is illustrated in Figure 4 . The successive 
rotations have increasing lengths and as a result, the two relaxed parts of the sample move apart from each other along the sample edge. Up to 5 rotations on each side may be observed successively. The propagation velocity of the rotated tear is quite slow, of the order of a few $\mathrm{mm} / \mathrm{s}$. The third step is the fast, catastrophic propagation starting from the last rotation, which leads to sample failure at a propagation rate of several $10 \mathrm{~s}$ of $\mathrm{m} / \mathrm{s}$. The final, catastrophic failure itself shows large deviations of the tear propagation direction (Figure 4, right), not observed in pure NR or in reinforced SBR. Such behavior was already described in filled natural rubber materials ${ }^{4}$.

Here we do not analyze in detail the stress (or strain) field near the crack tip, but rather propose a simple approach essentially based on energetic arguments. Specifically, we shall discuss and analyze quantitatively the effect of tear rotations on the ultimate strength of the samples.

Tear rotation enhances the tensile strength of the material, as suggested by Figure 2 and Figure 3 . Indeed, qualitatively, there seems to be a correlation between the measured energy at break and the overall length of the tear rotations: the longer the rotations, the higher the energy at break. Thus, developing long (or numerous) tear rotations allows a given sample to store more energy before it breaks, and the scattering of results obtained in reinforced NR samples (Figure 2 and Figure 3 ) is related to various values of the overall tear rotation length observed for a given value of $c$. Successive tear rotations occur as the sample is submitted to larger and larger elongation ratios (or to larger and larger stress levels).

We propose the following analysis for the relationship between tear rotation and ultimate strength of reinforced natural rubber. After a rotation has occurred, the tear tip becomes apparently flat in the deformed state. However, when back in the relaxed state (after failure of the sample), rotations take the form of paraboloid curves with the convexity directed towards the direction of the tear tip (see Figure 5). The part of the sample located inside the concavity of the rotation is relaxed. This situation is similar to having a cut with a finite, macroscopic radius $\rho$ in the relaxed state (not to be confused with the opening radius at an infinitely sharp tip), as described by Thomas ${ }^{23}$ and as studied also by Glucklich and Landel ${ }^{3}$.

Assuming a quasi- circular shape of the tear rotation in the relaxed state, the radius $\rho$ is related to the measured rotation length $l_{R}$ by $l_{R} \cong \pi \rho$ (see Figure 5 ). Thomas has shown the following approximate relationship between the energy release rate $G$ and the radius $\rho^{23}$ (for a macroscopic radius $\rho)$ :

$$
\text { 4. } G \approx W_{l o c} \rho
$$

where $W_{\text {loc }}$ is the elastic energy density at the tear tip, which is amplified with respect to the energy density far from the tip due to stress concentration. The smaller the tip radius, the higher the stress amplification. Thus, taking $W_{l o c} \cong W_{f}$, where $W_{f}$ is the energy density at failure of the material, as the criterium for failure at the tip, equation 4 effectively corresponds to an increase of the measured value of the elastic energy density at break $W_{b}$ as $\rho$ increases. Then, identifying the energy release rate (or tearing energy) $G$ in equation 4 to the same quantity defined in equation 2 gives the following relationship:

$$
\text { 5. } 2 \pi \frac{W_{b} c}{\sqrt{\lambda_{b}}}=W_{f} \frac{l_{R}}{\pi}
$$

This equation relates the ultimate property of a sample, through the quantity $2 \pi c W_{b} / \sqrt{\lambda_{b}}$ (which is homogeneous to a tearing energy, expressed in $\mathrm{kJ} . \mathrm{m}^{-2}$ ), to the length of the last rotation $I_{R}$. The quantity $2 \pi c W_{b} / \sqrt{\lambda_{b}}$ is plotted in Figure 6 and Figure 7 as a function of the total rotation length $L_{R}$ for experiments done on notch tensile strips with various precut 
notch length values, stretched at different traction speeds and temperatures, in samples reinforced with carbon black and silica, respectively. Note that the total length $L_{R}$ of all rotations. It is however largely dominated by the length of the ultimate rotation. The horizontal line in Figure 6 and Figure 7 corresponds to the value of the tearing energy $31.4 \mathrm{~kJ} . \mathrm{m}^{-2}$. The linear curves in both figures correspond to equation 5 . The slopes (denoted $W_{f} / \pi$ in equation 5 ) have been tuned to match the data qualitatively. The corresponding values correspond to $W_{f}=100 \mathrm{MPa}$ and $W_{f}=150 \mathrm{MPa}$ for carbon blackand silica-filled samples, respectively. These values are only semi-quantitative, and we shall not discuss them in terms of differences between both types of samples. Nevertheless, it is important to notice that these values are larger than the energy at break measured in the uncut samples, as expected (see

Table 2).

Thus, Figure 6 and Figure 7 show that equation 5 is semi-quantitatively verified, or at least gives the right order of magnitude for the relationship between the ultimate property (as expressed by the quantity $2 \pi c W_{b} / \sqrt{\lambda_{b}}$ ) and the rotation length. The large apparent scattering of points is mainly related to the fact that points obtained in different experimental conditions are plotted together. This allows one to see that temperature seems to have an effect on tear rotations. Tear rotations tend to be longer on average at low temperature. In carbon black-filled samples, rotation lengths $L_{R}$ measured in samples stretched at $-20^{\circ} \mathrm{C}, 0^{\circ} \mathrm{C}$ and $10^{\circ} \mathrm{C}$ are between $2 \mathrm{~mm}$ and $9 \mathrm{~mm}$, whereas those measured at higher temperatures $\left(40^{\circ} \mathrm{C}\right.$ and above) are between $0.6 \mathrm{~mm}$ and $3 \mathrm{~mm}$ typically. In silica-filled samples, rotation lengths measured at $40^{\circ} \mathrm{C}$ and above are between $2 \mathrm{~mm}$ and $10 \mathrm{~mm}$, those at $20^{\circ} \mathrm{C}$ are of the order $1 \mathrm{~mm}$.

The energy density at break in un-notched samples does not depend significantly on temperature, in the range $-20^{\circ} \mathrm{C}$ to $80^{\circ} \mathrm{C}$. On the other hand, the energy at break measured in precut samples does depend on temperature, as illustrated in Figure 8. From the previous discussion, this effect may be entirely due to the variation of the average tear rotation length as a function of temperature. Note also that, at comparable energy at break, the overall rotation length tends to be larger in samples stretched at low traction speeds.

It is clear from Figure 6 and Figure 7 that the data show a tendency to deviate from the linear variation expressed in equation 5 . This deviation may be interpreted semi-quantitatively in the following way. For a tear of length $c$ and finite tip radius $\rho$ (in the relaxed state), from standard elasticity in the linear regime, the elastic stress remains finite at the tip and is given by:

$$
\text { 6. } \sigma_{t} \approx\left(1+2\left(\frac{c}{\rho}\right)^{1 / 2}\right) \sigma_{0}
$$

where $\sigma_{0}$ is the stress in the homogeneously stretched part of the sample, far away from the tear tip. In terms of energy density (taking an energy density quadratic in the stress), this writes:

$$
\text { 7. } W_{l o c} \approx\left(1+2\left(\frac{c}{\rho}\right)^{1 / 2}\right)^{2} W_{0}
$$

$W_{0}$ is the elastic energy density far from the tip. Note that in general $\rho$ is not small compared to $c$ here. Assuming again that initiation of propagation (which corresponds to the detected onset of the next rotation) will occur when the local energy density $W_{\text {loc }}$ reaches some characteristic energy density at failure of the material $W_{f}$ leads to the relationship:

$$
\text { 8. } W_{b} \approx\left(1+2\left(\frac{c}{\rho}\right)^{1 / 2}\right)^{-2} W_{f}
$$

Assuming that the contour length $I_{R}$ of the rotation (in the relaxed state) is proportional to its radius in the relaxed state (typically $l_{R} \approx \pi \rho$ ), in the same way as before, leads to: 


$$
\text { 9. } W_{b} \approx\left(1+\kappa\left(\frac{c}{l_{R}}\right)^{1 / 2}\right)^{-2} W_{f}
$$

In which $\kappa$ is a geometrical, dimensionless parameter of order $2 \sqrt{\pi}$.

Actually, equation 9 (as well as equation 5) gives a relationship between the radius of the crack (related to the length $I_{R}$ of a given rotation, say rotation number $n$ ) and the onset of crack instability $W_{b}(n+1)$, assumed to correspond to initiation of the next tear rotation (rotation number $n+1$ ) or to the onset of propagation leading to final break of the sample.

An example of stress-strain curve obtained in a precut sample up to failure, in which the onsets of successive tear rotations have been determined by video monitoring, is shown in Figure 9. The onsets of successive tear rotations are indicated on the curve, together with the length (in $\mu \mathrm{m}$ ) of the corresponding rotation. The onset of strain induced crystallization in the bulk (non precut) sample, as detected by X-ray scattering, is also indicated on the curve. It is interesting to note that the first rotation occurs below the crystallization threshold. Nevertheless, due to local strain amplification, the material crystallizes in the vicinity of the tear tip quite early ${ }^{24}$, which should induce early rotation of the tear. Another point to note is that no large scale artifact occurs on the stress strain curve when a rotation appears and propagates.

Data illustrating equation 9 are shown in Figure 10 for samples with a precut notch length of the order $2 \mathrm{~mm}$. The threshold at initiation of a given rotation (corresponding to the quantity $W_{b}(n)$ in equation 9$)$ is plotted as a function of the length of the previous rotation $\left(I_{R}(n-1)\right.$ in equation 9$)$. The values of the parameters which have been used here are $W_{f}=100 \mathrm{MPa}$ and $\kappa=2.0$.

The correlation between the energy at break and the rotation length is also illustrated in Figure 11 and Figure 12, which show the elastic energy density at break $W_{b}$ as a function of the reduced total rotation length $L_{R} / c$, in samples reinforced with carbon black and silica, respectively. Results obtained at different values of the traction speed and temperature, corresponding to the same data as in Figure 6 and Figure 7, are shown. The curves in Figure 11 and Figure 12 correspond to equation 9. The following sets of parameters have been used to match the data: silica-filled samples: $W_{f}=100$ $\mathrm{MPa}, \kappa=1.5$; carbon black-filled samples: $W_{f}=100 \mathrm{MPa}, \kappa=1.75$.

Finally, data obtained at room temperature and a tensile rate of $0.014 \mathrm{~s}^{-1}$ are shown in Figure 13 for samples reinforced with carbon black and silica. Both sets of data have been matched with equation 9, using for each type of sample the same set of parameters as in Figure 11 and Figure 12, that is: silica-filled samples: $W_{f}=100 \mathrm{MPa}, \kappa=1.5$; carbon black-filled samples: $W_{f}=100 \mathrm{MPa}$, $\kappa=1.75$. Silica-filled samples tend to show slightly longer rotations than CB-filled samples.

\section{Discussion}

For the first time, a mechanism is proposed to relate the presence of tear rotations observed in reinforced natural rubber to the tensile strength of the material. The mechanism which we propose to describe the tear rotation pattern is the following one: when the test sample is stretched at a given extension rate, the precut notch opens widely without visible propagation, as long as the local energy density at the tear tip $W_{l o c}$ remains below a critical value $W_{f}$ for initiation of propagation. When $W_{\text {loc }}=W_{f}$, propagation initiates. After propagating over a few 10 's of $\mu \mathrm{m}$, the tear rotates. Tear rotation effectively releases part of the stress amplification at the tear tip, by creating a macroscopic 
tear tip radius $\rho$ (which scales as and is comparable to the rotation length). This may provide a purely elastic mechanism for effective tip blunting, distinct from the Dugdale-Barenblatt visco-plastic mechanism ${ }^{25,26}$. In the rotated tear configuration, the relationship between the macroscopic energy density $W_{0}$ (far away from the tear) and the local energy density at the tear tip $W_{\text {loc }}$ is given by equation 7. Then the test sample can be further stretched, until the local energy density at the tear tip reaches again the critical value $W_{f}$. Thus, in this proposed mechanism, each successive rotation effectively results in a larger tip radius, which allows reaching larger macroscopic values of the elastic energy density in the bulk of the sample, according to equation 7.

Equations 6 and 7 and the resulting equation 9 have been written by assuming linear elasticity, which of course is not valid in rubbers at the very large local strain values considered here. However, the analysis proposed here is only semi quantitative. The data have a quite large statistical scattering whatsoever. Taking non-linearities into account would not change the analysis and the conclusions. The proposed mechanism allows matching the data quite well with equation 9, which contains two parameters: the local energy density at onset of propagation (denoted $W_{f}$ ), which is coherently larger than the tensile strength of bulk (non precut) samples; a geometrical factor $\kappa$. The value of this factor would certainly be affected by non linearities, but its order of magnitude should essentially be preserved.

The tear rotation phenomenon observed here and illustrated in Figure 4 has some similarities with the transition between cohesive and interfacial failure in a laminate described by Kendall ${ }^{27}$. Both situations are different, however. Kendall describes a situation in which a crack propagating in a material (in mode I) meets a perpendicular interface, and examines the conditions under which the crack may be deflected along this weaker interface. In the present case, a weaker interface (characterized by a given cohesive energy) does not preexist in the material. Such an interface must therefore be created within the material during the crack propagation itself. This phenomenon has been attributed to the occurrence of strain-induced crystallization in NR and in filled NR $28,29,31,32$. Indeed, it has been shown that there is a crystallized region in front of the tear tip ${ }^{18},{ }^{24}$. The extension of this region, related to strain concentration at the tear tip, depends on the overall extension ratio (far from the tear tip) and becomes infinite at the onset of bulk crystallization. It is likely that the presence of this crystallized region prevents the tear to propagate forwards in the normal direction (perpendicular to the direction of extension) because it induces a strong elastic anisotropy in the material, which would be the main mechanism responsible for tear rotation.

However, strain induced crystallization by itself is not sufficient to induce tear rotation, as pure NR samples (which do crystallize) do not exhibit tear rotation. A combination of strain-induced crystallization and of reinforcement provided by nanometric fillers, which both shift the onset of crystallization to lower overall strain and considerably increase the modulus, is thus needed to induce this phenomenon. Also, it is worth mentioning that tear rotation does not appear in the absence of a precut notch (of millimeter size).

It is observed that the material exhibits well developed fibrillar morphology at very high extension ratios. Then it is likely that such fibrils have a very high modulus (that of a semi-crystalline polymer) in the presence of strain-induced crystallization, thus providing the strong anisotropy in the elastic properties needed to observe tear rotation. Indeed, such anisotropy could favor propagation along the tensile direction, as compared to normal propagation (perpendicular to tensile direction). 
However, the anisotropy in local elastic properties has not been quantified (equivalent to the ratio of cohesive vs adhesive energies as discussed by Kendall) and thus the conditions for onset of tear rotation is not discussed here.

The argument developed in Section 3.2 does not apply to the first rotation, which initiates in the tip of the precut notch itself (with a micrometric tip radius). The occurrence and size of the first rotation may be analyzed as follows, using qualitative arguments based on linear elasticity. For a cut with an infinitely sharp tip, standard linear elasticity gives a parabolic opening of the tear tip, with an opening radius in the deformed state (not to be confused with the finite tip radius created through tear rotation, denoted $\rho$ previously):

$$
\text { 10. } R=\frac{1}{4 \pi}\left(\frac{3 K}{E}\right)^{2} \approx \frac{3}{4} \frac{\sigma_{0}{ }^{2} c}{E^{2}} \approx \frac{3}{2} \frac{W c}{E}
$$

In which $K=\sigma_{0} \sqrt{\pi c}$ is the stress intensity factor (in opening mode I), $E$ is the Young's modulus, and $W=\sigma_{0}{ }^{2} / 2 E$ is the strain energy density far from the tear. The Poisson ratio has been taken to be $v=0.5$, the value for an incompressible material. Now we make the assumption that the length $I_{R}$ of the first rotation scales as, and is of the order of, the tip opening radius $R$ (perhaps within a geometrical factor), which is the only characteristic length scale at the tear tip in the deformed state (neglecting finite size effects). According to equation 10 , it follows that $W$ is proportional to $I_{R}$ :

$$
\text { 11. } l_{R} \approx \frac{W c}{E}
$$

The first rotation occurs at a strain energy density of the order $\mathrm{W} \approx 0.2$ to $0.4 \mathrm{MPa}$ with the precut notch length $c=2$ $\mathrm{mm}$ (see Figure 9). The modulus is of the order $3 \mathrm{MPa} \mathrm{Pa}$ (see

Table 2). Equation 11 then gives $l_{R} \approx 0.15$ to $0.3 \mathrm{~mm}$, which is indeed exactly the right order of magnitude, as indicated in Figure 10, which shows that the observed length of the first rotation is effectively between 0.2 and $0.3 \mathrm{~mm}$. Note that this argument relates $W$ to $I_{R}$ for the first rotation, but does not give the value of the onset of propagation for this rotation. The value of the onset for a given precut notch length $c$ is rather given by equation 3 .

Finally, tear rotations have little effect on the stress-strain curves, as it is illustrated in Figure 9. This is very different from the large oscillations of the tearing force which are observed e.g. in tearing tests performed in the trouser geometry ${ }^{33}$. Only in some cases, with very large tear rotations, stressstrain curves exhibit some visible accidents, which are very much reminiscent of the large oscillations of the tearing force observed in the regime of so-called knotty tearing in the trouser test geometry. The drop in the force corresponds to forward tear propagation. Then, as tear deviates from its normal direction, the force start to increase again, as propagation along the direction of extension corresponds to a vanishing energy release rate.

We have compared NR samples reinforced with carbon black and precipitated silica. Both types of samples were formulated in order to have similar crosslink densities, filler volume fractions, and altogether have quite similar mechanical properties. Both types of samples show very similar behavior as regards the development of tear rotation and resulting tensile strength.

\section{Conclusion}

For the first time, a mechanism is proposed to relate the presence of tear rotations observed in reinforced natural rubber to the tensile strength of the material. The measured apparent tensile 
strength (in terms of energy at break) may be increased by a factor of 6 to 8 in some cases. This large increase in tensile strength associated to the presence of tear rotations is analyzed semiquantitatively, based on energetic arguments, without entering into a detailed description of the elastic strain field in the vicinity of the tear tip. We show a correlation between the extent of the phenomenon of tear rotation and the energy density at failure of single edge notched tensile test samples. We also show a correlation between the length of a rotation and the stress level at which it appears. The proposed interpretation is based on the idea that tear rotations relax the local strain (or stress) at the tear tip by creating a macroscopic tip radius. This is a mechanism of tip blunting which is different from visco-plastic effects described first by Dugdale and Barenblatt.

\section{Bibliography}

1. Greensmith HW. Rupture of Rubber. IV. Tear Properties of Vulcanizates Containing Carbon Black. Journal of Polymer Science. 1956;21:175-187.

2. Greensmith HW. Rupture of Rubber. VIII. Comparisons of Tear and Tensile Rupture Measurements. Journal of Applied Polymer Science. 1960;3(8):183-193.

3. Glucklich J, Landel RF. The tearing test as a mean fo estimating the ultimate properties of rubber. Journal of Applied Polymer Science. 1976;20:121-137.

4. Hamed GR, Kim HJ, Gent AN. No Title. Rubber Chemistry and Technology. 1996;69:807.

5. Lake GJ, Samsuri A, .Teo SC, Vaja J. Time-dependent fracture in vulcanized elastomers. Polymer. 1991;32(16):2963-2975.

6. Busse WF. Tear resistance and structure of rubber. Ind. Eng. Chem. 1934;26.

7. Stacey R, Yanyo L, Kelley F. Observations on the tearing of elastomers. Rubber Chem. Tech. 1986;58:421.

8. Thomas AG, Whittle JM. No Title. Rubber Chemistry and Technology. 1970;43:222.

9. Hamed GR, N. Rattanasom. Effect of crosslinking density on cut growth in black-filled natural rubber vulcanizates. Rubber Chemistry and Technology. 2002;75:935-941.

10. Kim HJ, Hamed G. No Title. Rubber Chemistry and Technology. 2000;73:743.

11. Hamed G, Park BH. No Title. Rubber Chemistry and Technology. 1999;72:946.

12. Hamed G, Kim HJ. No Title. Rubber Chemistry and Technology. 1999;72:895.

13. Hamed G, Huang MY. No Title. Rubber Chemistry and Technology. 1998;71:846.

14. Hamed G, Zhao J. No Title. Rubber Chemistry and Technology. 1998;71:157. 
15. Hamed G, N. Rattanasom. Effect of density on cut growth in gum natural rubber vulcanizates. Rubber Chem. Tech. 2002;75:323.

16. Hamed G, Al-Sheneper AA. No Title. Rubber Chemistry and Technology. 2003;76:436.

17. Andrews EH. Crack Propagation in a Strain-Crystallizaing Elastomer. Journal of Applied Physics. 1961;32:542-548.

18. Lee DJ, Donovan JA. Microstructural changes in the crack tip region of carbon-black-filled natural rubber. Rubber Chemistry and Technology. 1987;60:910-923.

19. Persson BN, Albohr O, Heinrich G, Uebas H. Crack propagation in rubber-like materials. JOURNAL OF PHYSICS: CONDENSED MATTER. 2005;17:R1071 R1142.

20. Lake GJ, Lawrence CC, Thomas AG. High-speed fracture of elastomers:part I. Rubber Chemistry and technology. 2000;73:801-817.

21. Rivlin RS, Thomas AG. No Title. J. Polym. Sci. 1953;10:291.

22. Lake GJ. No Title. In: Proc. Int. Conf. on Yield, Deformation and Fracture of Polymers, Cambridge.; 1970:5.3/1.

23. Thomas AG. Rupture of rubber. II. The strain concentration at an incision. Journal of Polymer Science. 1955;18:177-188.

24. Trabelsi S, Albouy P, Rault J. Stress-Induced Crystallization around a Crack Tip in Natural Rubber. Macromolecules. 2002;35:10054-10061.

25. Dugdale SD. No Title. J. Mech. Phys. Solids. 1960;8:100.

26. Barenblatt GI. No Title. Adv. Appl. Mech. 1962;7:55.

27. Kendall K. Transition between Cohesive and Interfacial Failure in a Laminate. Proc. R. Soc. Lond. A. 1975;344:286-302.

28. Gent AN. No Title. Trans. Faraday Soc. 1954;50:521.

29. Miyamoto Y, Yamao H, Sekimoto K. Crystallization and melting of polyisoprene rubber under unixial deformation. Macromolecules. 2003;36:6462-6471.

30. Poompradub S, Tosaka M, Kohjiya S, et al. No Title. J. Appl. Phys. 2005;97:103529.

31. Chenal J, Gauthier C, Chazeau L, Guy L, Bomal Y. Parameters governing strain induced crystallization in filled natural rubber. Polymer. 2007;48:6893-6901.

32. Rault J, Marchal J, Judeinstein P, Albouy PA. Stress-Induced Crystallization and Reinforcement in Filled Natural Rubbers: 2H NMR Study. Macromolecules. 2006;39:8356-8368. 
33. Stacer RG, Von Meerwall ED, Kelley FN. Time-dependent tearing of carbon black-filled and strain crystallizing vulcanizates. Rubber Chemistry and Technology. 1986;58:913. 


\section{Tables}

\begin{tabular}{|c|cc|cc|c|c|}
\hline Formulation (phr) & SBR-s & SBR-c & NR-s & NR-c & SBRO & NRO \\
\hline S-SBR 2525-0 & 100 & 100 & & & 100 & \\
NR CV60 & & & 100 & 100 & & 100 \\
Silica (Z 160 MP) & 50 & & 50 & & & \\
Carbon black (N234) & & 45 & & 45 & & \\
TESPT $^{2}$ & 4 & & 4 & & & \\
6PPD $^{3}$ & 1.9 & 1.9 & 1.9 & 1.9 & 1.9 & 1.9 \\
Stearic acid & 4 & 4 & 4 & 4 & 4 & 4 \\
ZnCO $_{3}$ & 4.6 & 4.6 & 4.6 & 4.6 & 4.6 & 4.6 \\
Sulfur $_{\text {CBS }}^{4}$ & 1.5 & 1.5 & 1.5 & 1.5 & 1.5 & 1.5 \\
TBzTD $^{5}$ & 1.7 & 1.7 & 1.7 & 1.7 & 1.7 & 1.7 \\
Curing time (min) & 0.24 & 0.24 & 0.24 & 0.24 & 0.24 & 0.24 \\
\hline
\end{tabular}

Table 1. Sample formulations. 1: precipitated silica, surface $160 \mathrm{~m}^{2} \mathrm{~g}^{-1} .2$ : bis triethoxysilylpropyltetrasulfur: provides covalent coupling between the matrix and silica particles. 3: $\mathrm{N}$-(1,3-dimethylbutyl)- $\mathrm{N}$ '-phenyl-P-phenylenediamine, used as antioxidant. 4: $\mathrm{N}$-cyclohexyl-2benzothiazyl, used as primary accelerator. 5: tetrabenzylthiuram disulfide, used as secondary accelerator. 


\begin{tabular}{|c|c|c|c|}
\hline Sample & $\begin{array}{c}\text { Nominal stress at } \\
\text { break (MPa) }\end{array}$ & $\begin{array}{c}\text { Energy density } \\
\text { at failure } W_{f} \\
\text { (MPa) }\end{array}$ & Modulus (MPa) \\
\hline SBRO & $1.85 \pm 0.5$ & 2 & 2.4 \\
SRO & 17.9 & 14.8 & 2.28 \\
SBR-c & 21.4 & 25.8 & 2.98 \\
NR-s & 29 & $50 \pm 5$ & 3.13 \\
NR-c & 31 & $55 \pm 5$ & 2.3 \\
\hline
\end{tabular}

Table 2. Mechanical data of the samples, measured in un-notched tensile strips strained at $0.014 \mathrm{~s}^{-1}$. The indicated modulus is the secant modulus (stress over strain ratio) measured in uniaxial traction (strain rate $0.014 \mathrm{~s}^{-1}$ ) at $100 \%$ strain. This is representative of the modulus at moderate to high extension ratios (beyond Payne effect). 


\section{Figures}

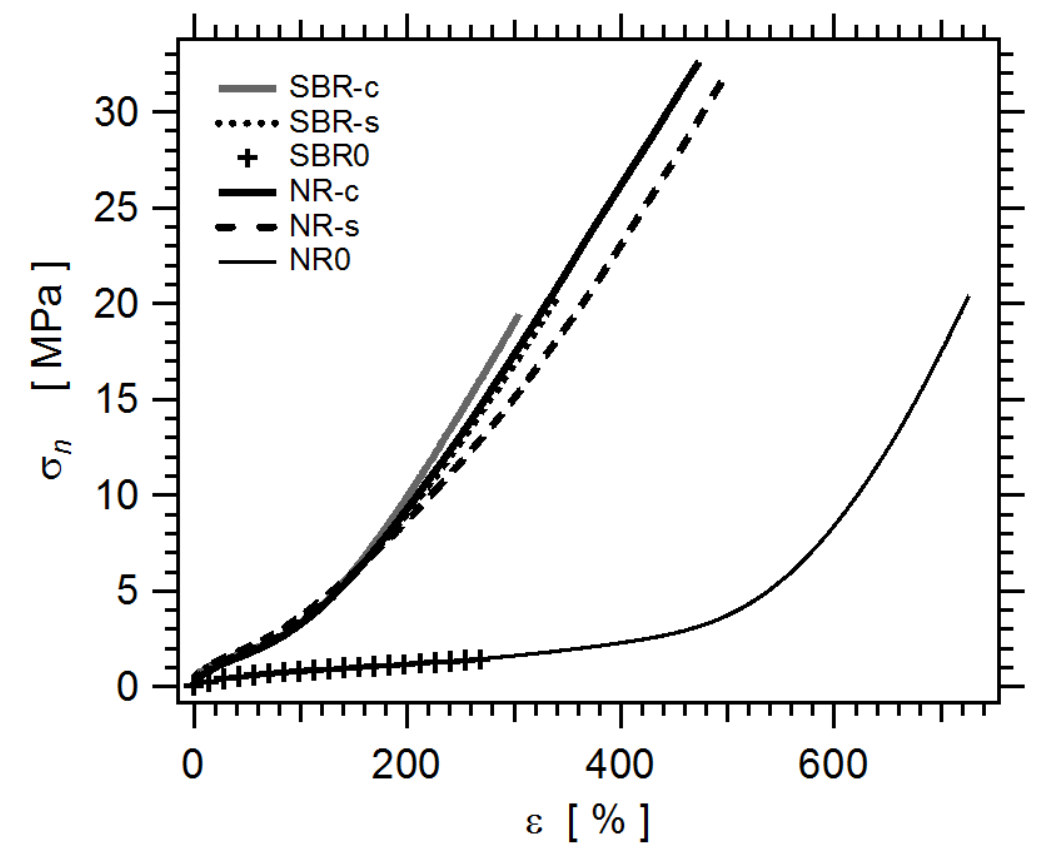

Figure 1. Nominal stress vs strain curves in the studied samples. End points of the curves correspond to tensile failure of the un-notched samples. 


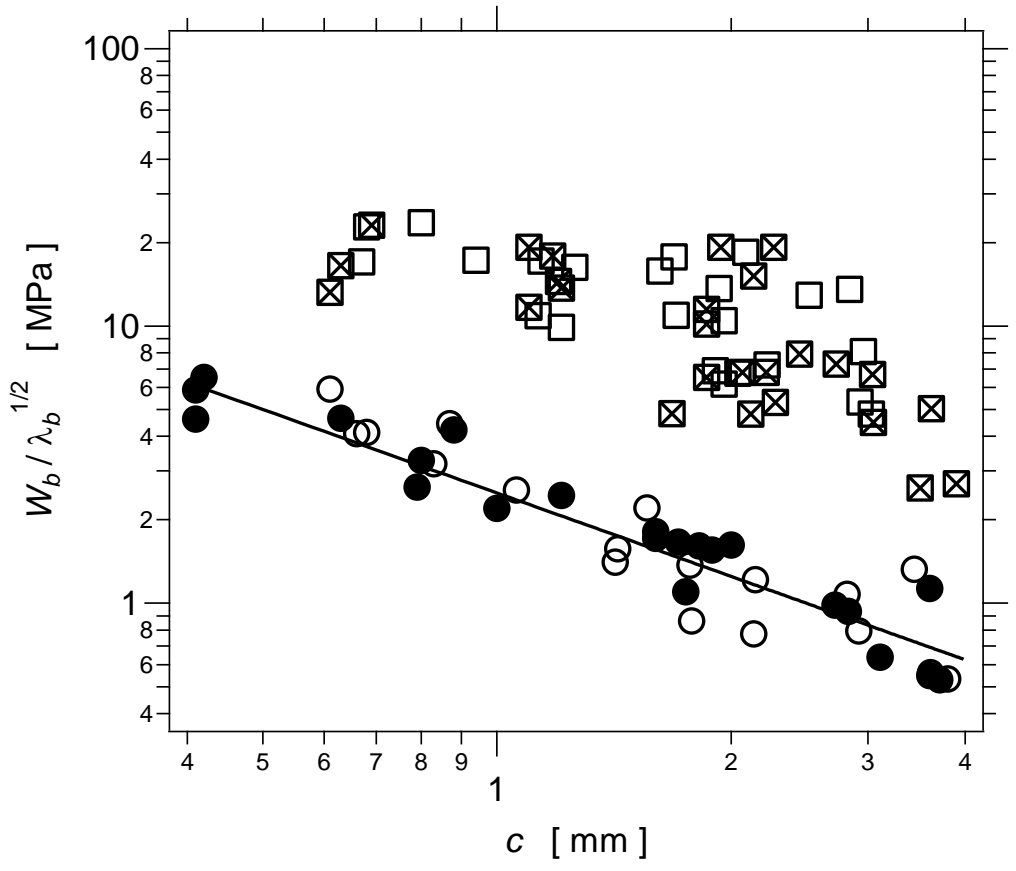

Figure 2 : The quantity $W_{b} / \sqrt{\lambda_{b}}$, where $W_{b}$ is the energy density at break and $\lambda_{b}$ the elongation ratio at break, as a function of the precut length $c$ for various samples: NR-s $(\square)$, NR-c $(\bigotimes)$, SBR-s $(0)$ and SBR-c $(\bullet)$. Temperature $T=22^{\circ} \mathrm{C}$. 


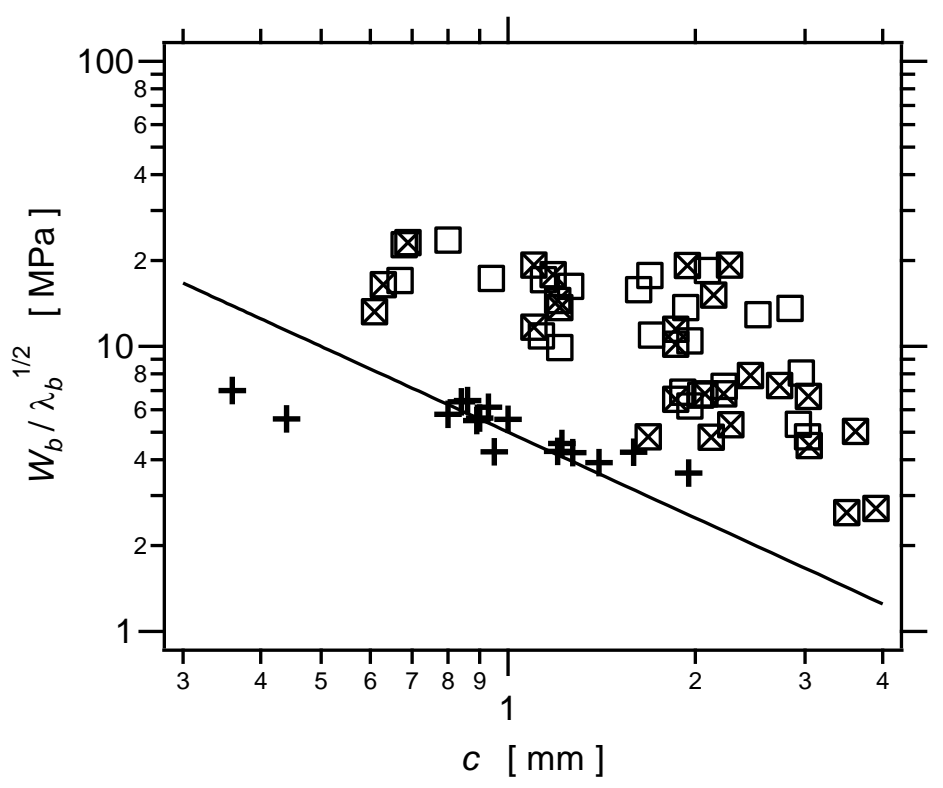

Figure 3 : The quantity $W_{b} / \sqrt{\lambda_{b}}$, where $W_{b}$ is the energy density at break and $\lambda_{b}$ the elongation ratio at break, as a function of the precut length $c$ for samples: NR-s ( $\square$ ), NR-c $(\bigotimes)$, NRO (pure NR) (+). Data for reinforced NR samples (NR-s and NR-c) are the same as in Figure 2. 

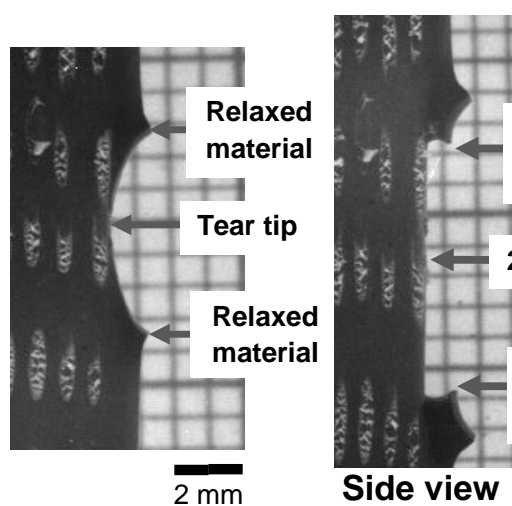

Tip of $1^{\text {st }}$ rotation

$2^{\text {na }}$ rotation

Tip of 1 st rotation

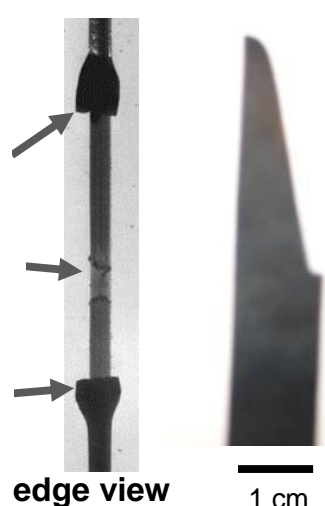

Figure 4 : Macroscopic observation of successive tear rotations in a precut notched reinforced NR tensile strip. On the right is shown one part of the sample after failure. Final, catastrophic propagation deviates strongly from normal direction. Note that some marks have been drawn of the sample surface. 
a

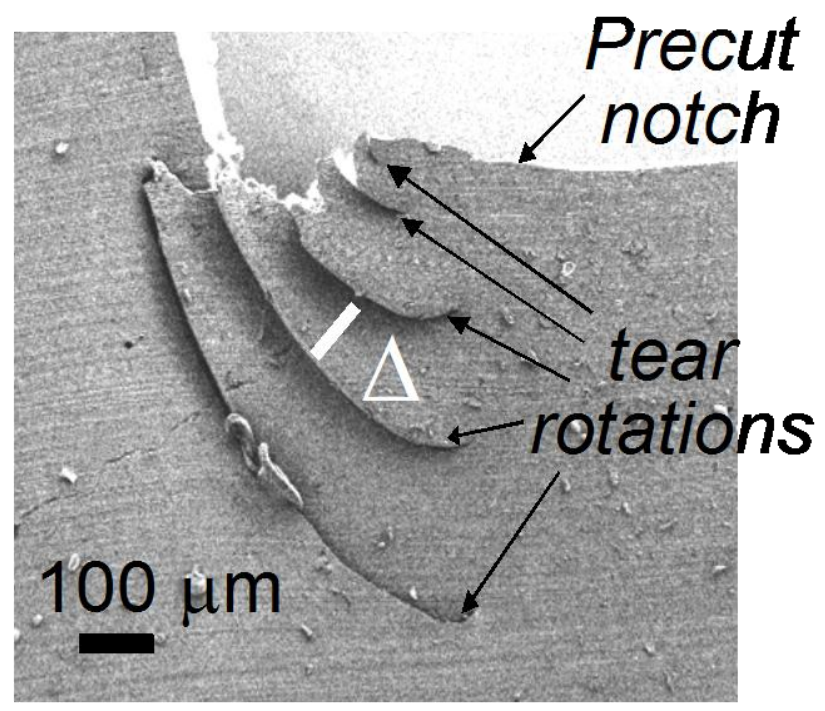

b
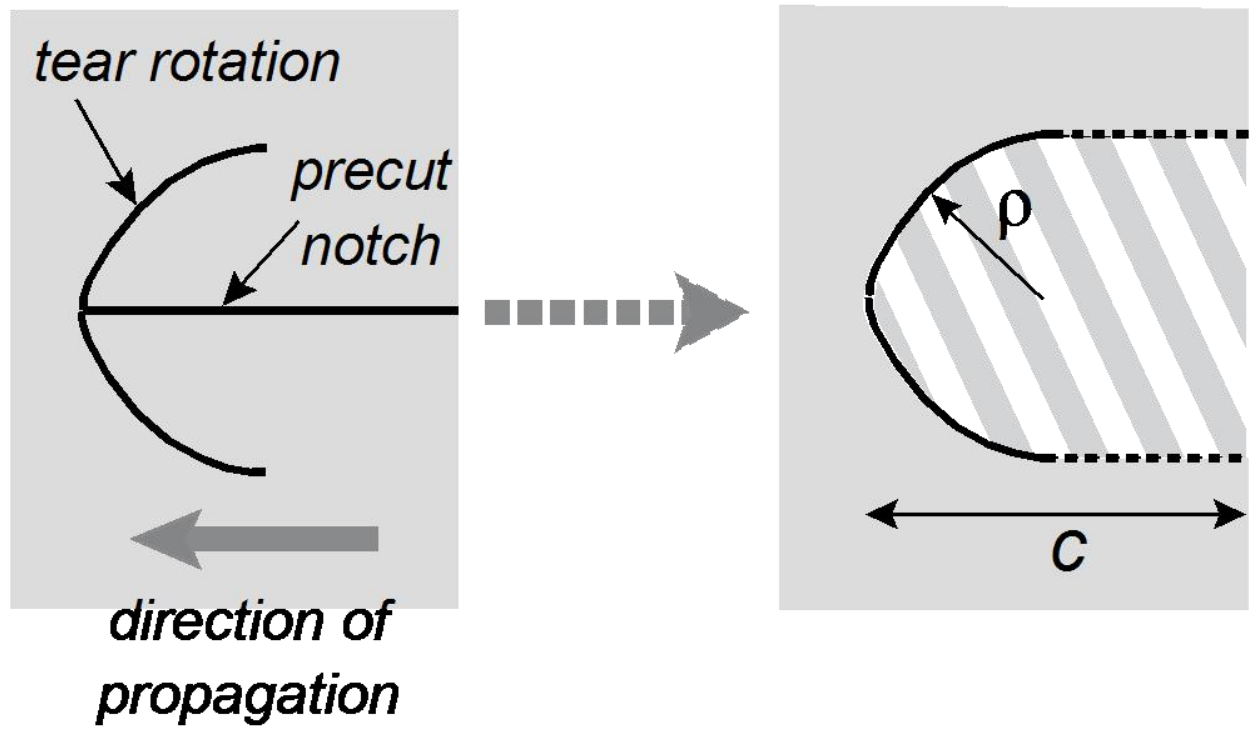

Figure 5. : a: post mortem MEB observation of rotations in a silica filled NR sample; $b$ : scheme illustrating tip blunting at a tear rotation, which effectively results in a notch of finite radius $\rho$. The curvilinear length $I_{R}$ of the rotation (measured in the relaxed state) is $l_{R} \cong \pi \rho$. 


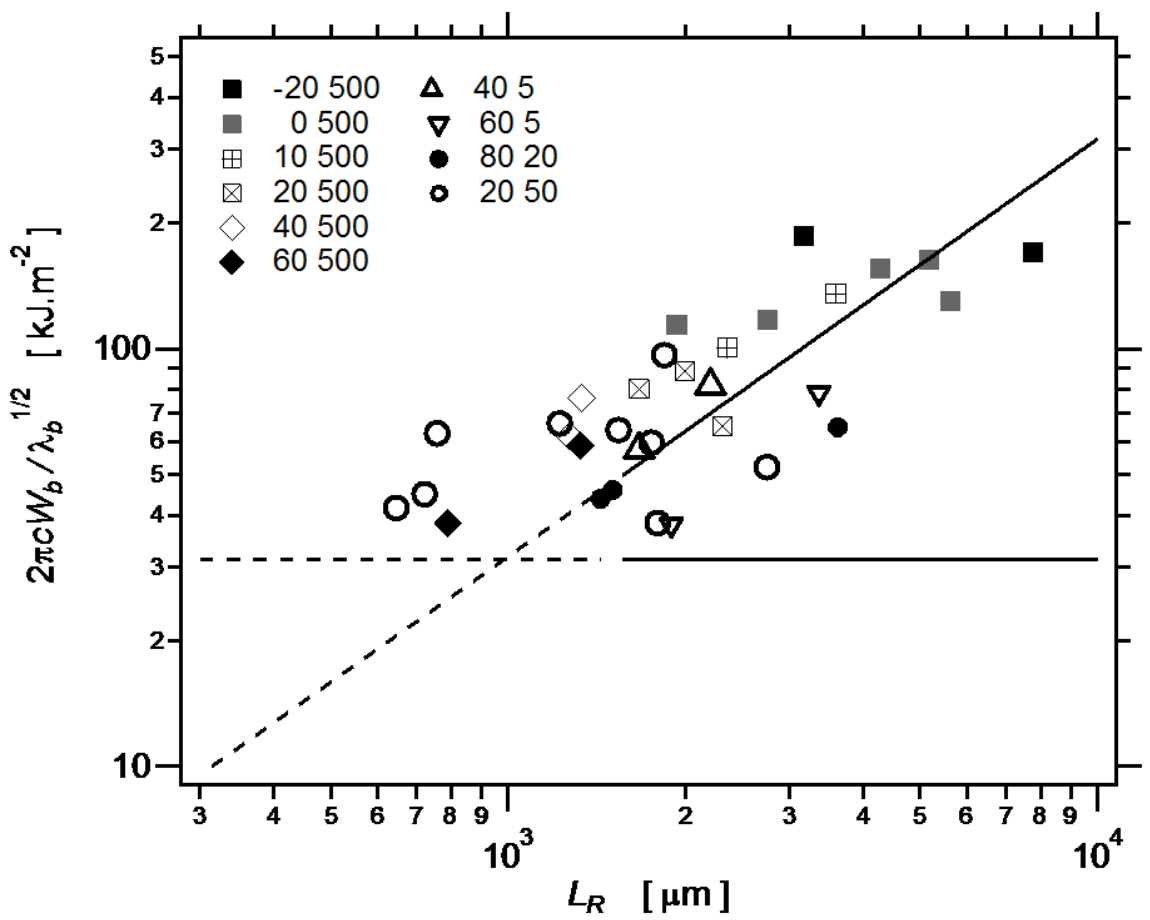

Figure 6. The quantity $2 \pi c W_{b} / \sqrt{\lambda_{b}}$ as a function of the rotation length $L_{R}$ in carbon black-filled samples, for various values of the traction speed, temperature and precut notch length. Values of the temperature in ${ }^{\circ} \mathrm{C}$ (first figure) and of the traction speed in $\mathrm{mm} / \mathrm{min}$ (second figure) are indicated in the legend. The horizontal line indicates the value 31.4 $\mathrm{kJ} . \mathrm{m}^{-2}$ obtained from the data in pure NR samples (see Figure 3). The linear curve corresponds to equation 5. 


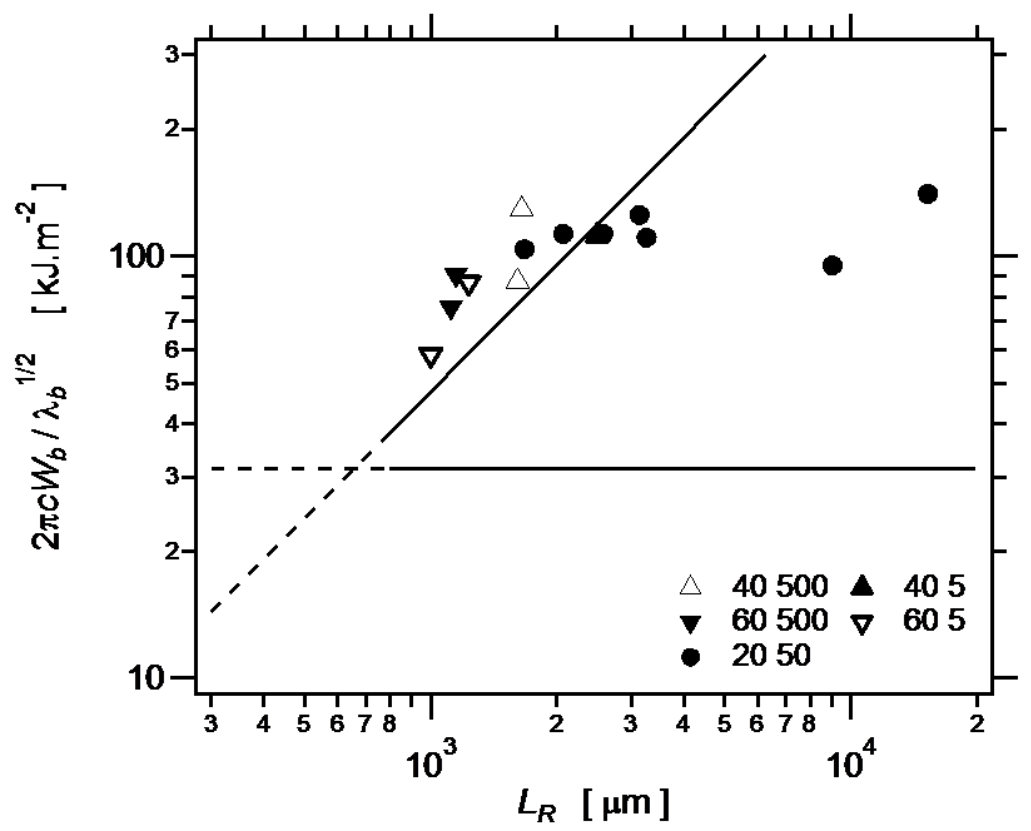

Figure 7. The quantity $2 \pi c W_{b} / \sqrt{\lambda_{b}}$ as a function of the total rotation length $L_{R}$ in silica-filled samples, for various values of the traction speed, temperature and precut notch length. . Values of the temperature in ${ }^{\circ} \mathrm{C}$ (first figure) and of the traction speed in $\mathrm{mm} / \mathrm{min}$ (second figure) are indicated in the legend. The horizontal line indicates the value 31.4 $\mathrm{kJ} . \mathrm{m}^{-2}$ obtained from the data in pure NR samples (see Figure 3). The linear curve corresponds to equation 5. 


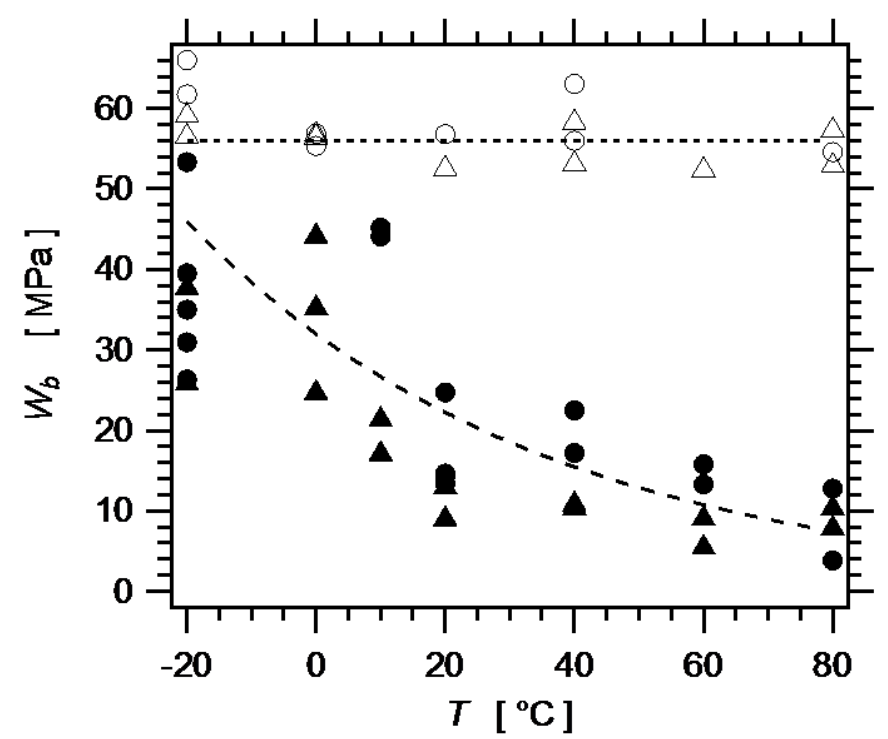

Figure 8. The energy density at break $W_{b}$ as a function of temperature. Triangles: carbon black-filled samples; circles/disks: silica-filled samples. Open symbols: non precut samples; filled symbols: samples with a precut notch length $c=2 \mathrm{~mm}$. Dashed/dotted curves are only guides to the eye. 


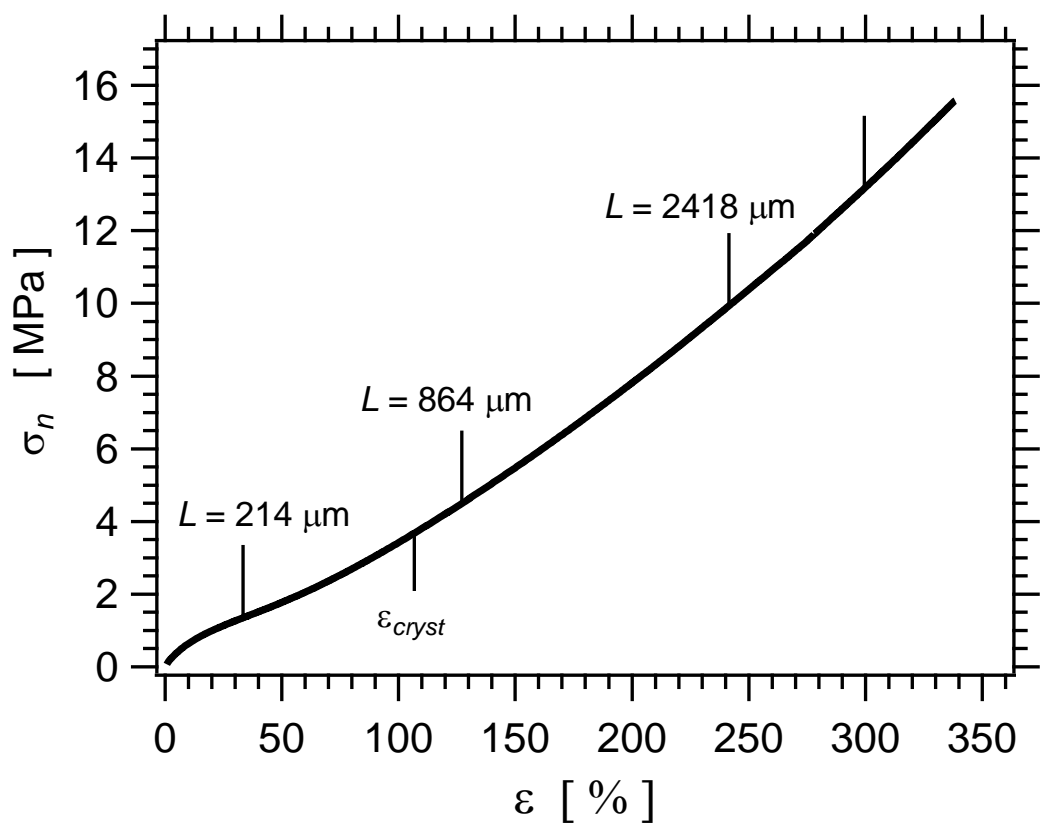

Figure 9 : The nominal stress $\sigma_{n}$ as a function of the macroscopic deformation in a precut Silica-filled NR sample stretched up to failure (precut notch length $c=2 \mathrm{~mm}$ ). The onsets of successive tear rotations are indicated, together with the length of the corresponding rotation. The last rotation initiates around $300 \%$ overall strain, it propagates along the applied extension until it leads to failure at about $340 \%$. The onset of strain-induced crystallization in the bulk $\varepsilon_{c r y s t}$ is also indicated. 


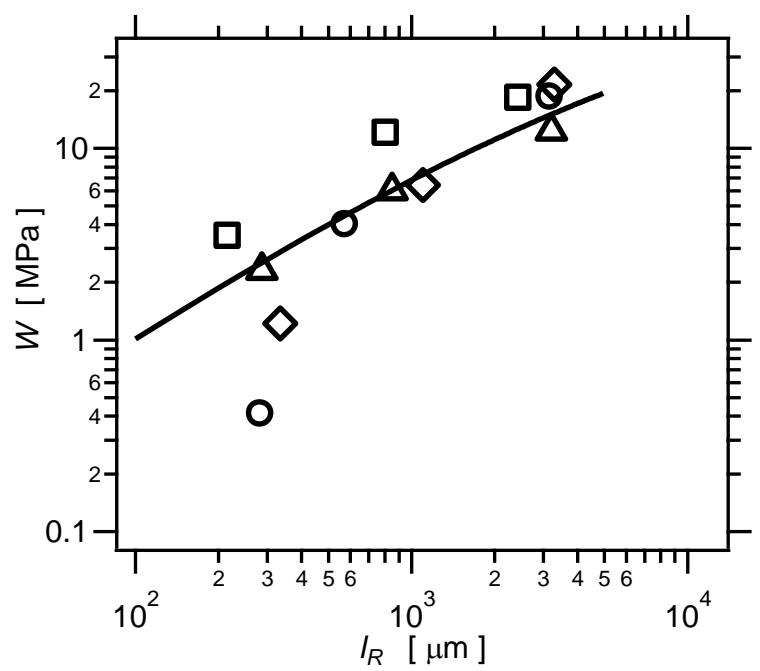

Figure 10. The elastic energy density $W(n+1)$ at the onset of tear rotation $n+1$ (for $n=0$ to 2 ), as a function of the length $I_{R}(n)$ of the previous rotation (which determines the effective tear tip radius), in silica-filled samples (NR-s) with a precut notch length $c=2 \mathrm{~mm}$. Each type of symbol corresponds to a particular test sample. For each sample, data were collected as indicated for one example in Figure 9. The curve corresponds to equation 9. 


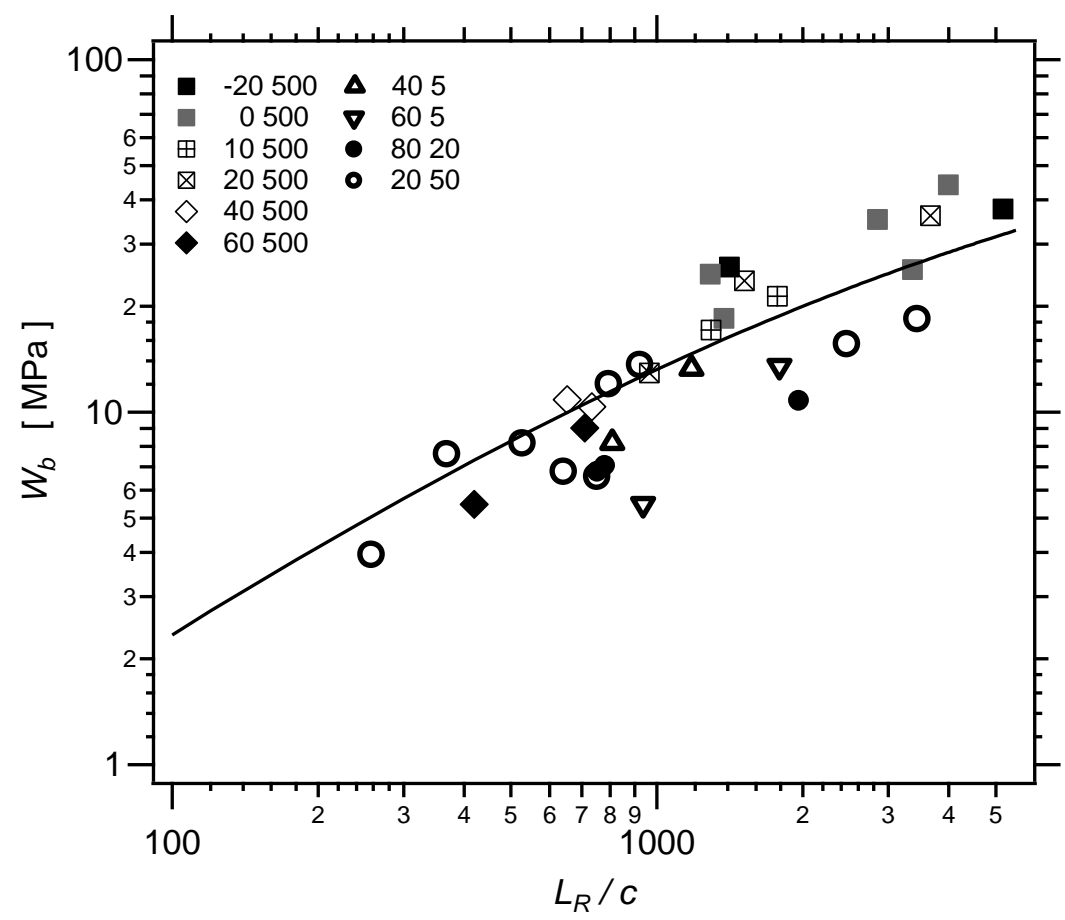

Figure 11 : The energy density at break $W_{b}$ as a function of the reduced total rotation length $L_{R} / c\left(L_{R}\right.$ is in $\mu \mathrm{m}, c$ in $\left.\mathrm{mm}\right)$ in carbon black-filled NR samples, stretched at various traction speeds and temperatures (from the same set of experimental data as in Figure 6). The curve corresponds to equation 9. 


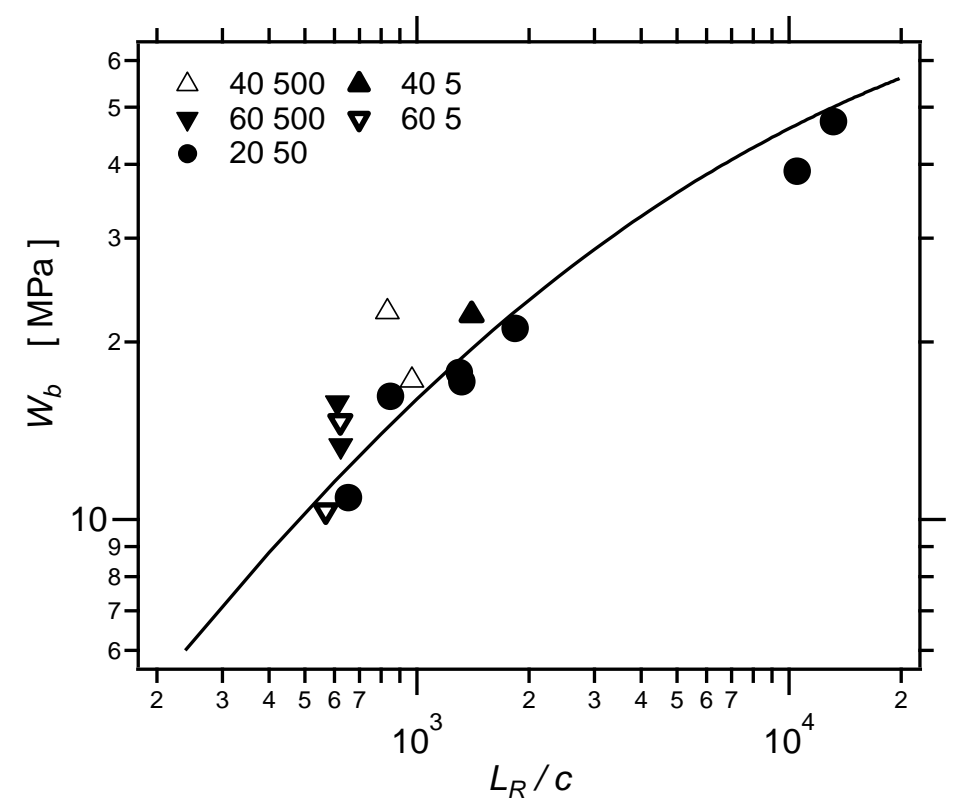

Figure 12. The energy density at break $W_{b}$ as a function of the reduced total rotation length $L_{R} / c\left(L_{R}\right.$ is in $\mu \mathrm{m}, c$ in $\left.\mathrm{mm}\right)$ in silica-filled NR samples, stretched at various traction speeds and temperatures (from the same set of experimental data as in Figure 7). The curve corresponds to equation 9. 


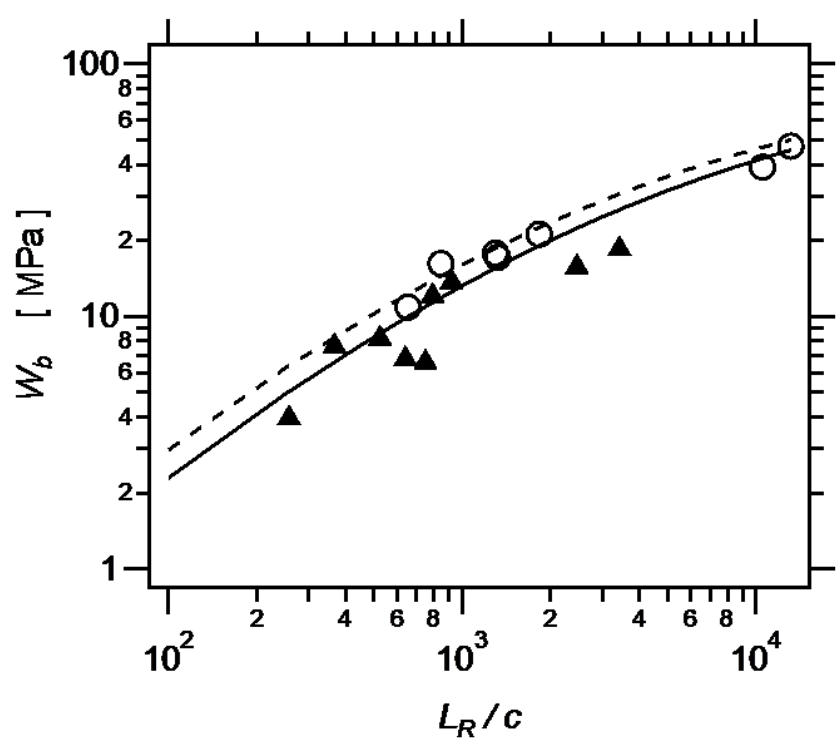

Figure 13. The energy density at break as a function of the reduced total rotation length $L_{R} / c\left(L_{R}\right.$ is in $\mu \mathrm{m}, c$ in $\left.\mathrm{mm}\right)$ in carbon black-filled (full triangles) and silica-filled (circles) samples, measured at strain rate $1.4 \times 10^{-2} \mathrm{~s}^{-1}$ and $T=22^{\circ} \mathrm{C}$. Curves are fits to equation 9, using the same sets of parameters as in Figure 11 and Figure 12: $W_{f}=100 \mathrm{MPa}, \mathrm{K}=1.5$ (silica-filled samples, dashed curve); $W_{f}=100 \mathrm{MPa}, \kappa=1.75$ (carbon black-filled samples, full curve). 


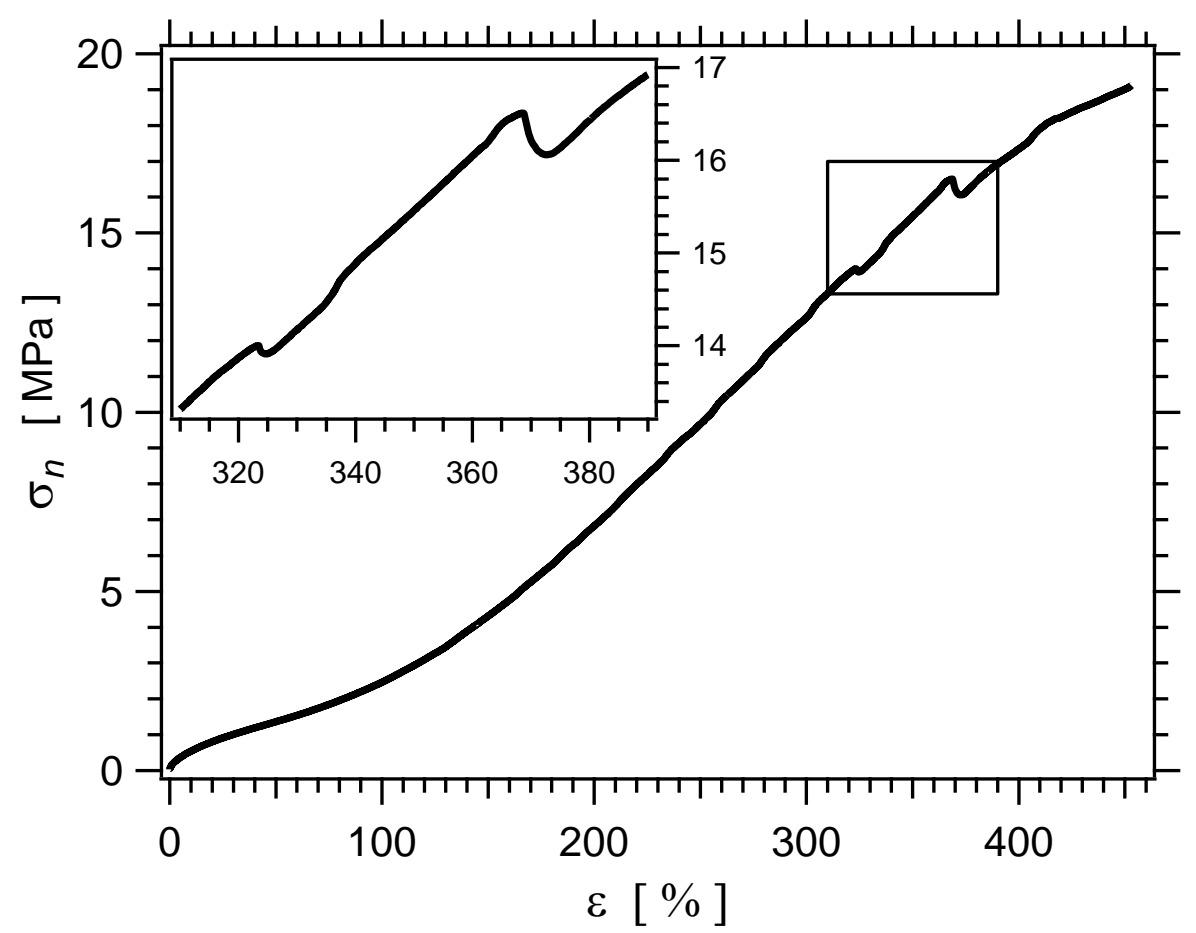

Figure 14. The nominal stress $\sigma_{n}$ as a function of the macroscopic deformation in a precut Silica-filled NR sample stretched up to failure (precut notch length $c=2 \mathrm{~mm}$ ). The inset emphasizes a region of the curve in which large tear rotations occur. 